\title{
Nutritional status is strongly correlated with grip strength and depression in community-living elderly Japanese
}

\author{
Tomoko Kaburagi i,* , Reiko Hirasawa ${ }^{2}$, Haruka Yoshino ${ }^{3}$, Yukino Odaka ${ }^{4}$, \\ Mariko Satomi ${ }^{4}$, Mana Nakano ${ }^{4}$, Eka Fujimoto ${ }^{4}$, Kazuyuki Kabasawa ${ }^{1}$ \\ and Kazuto Sato ${ }^{4}$ \\ 'Department of Health Science, Faculty of Sports and Health Sciences, Daito Bunka University, 560 Iwadono, \\ Higashi-matsuyama, Saitama 355-8501, Japan: ${ }^{2}$ Department of Internal Medicine, University of Tsukuba Institute \\ of Clinical Medicine, Ibaragi, Japan: ${ }^{3}$ Department of Health and Nutrition, Yamanashi Gakuin University, \\ Yamanashi, Japan: ${ }^{4}$ Department of Food and Nutrition, Japan Women's University, Tokyo, Japan
}

Submitted 19 August 2010: Accepted 27 January 2011: First published online 23 March 2011

\begin{abstract}
Objectives: To evaluate the utility of the Mini-Nutritional Assessment (MNA) in assessing nutritional, physical and psychosocial functions in community-living elderly individuals.

Design: A cross-sectional study of elderly individuals investigated in August 2007 and August-September 2008. Nutritional status was assessed using serum biomarkers, anthropometric measurements and the MNA. Physical function was assessed by measuring grip strength and both usual and maximum walking speeds. The Geriatric Depression Scale (GDS) was used to measure the individual's depressive state.

Setting: Elder-care facilities in Tokyo, Japan.

Subjects: Community-living elderly individuals aged $\geq 65$ years ( $n 130)$.

Results: The MNA evaluation classified twenty-seven (20.8\%) individuals as being at risk for malnutrition (MNA score $\leq 23 \cdot 5$ ); these at-risk individuals included a high proportion of the elderly aged $\geq 75$ years. MNA scores correlated with nutritional biomarkers (total protein, albumin, cholinesterase, $\mathrm{Hb}$ ) and anthropometric measurements (triceps skinfold, subscapular skinfold, mid-arm muscle area) and exhibited a strong correlation with grip strength and GDS score. Multivariate analysis revealed that grip strength, GDS score, marital status and maximum walking speed are strong predictors of MNA score.

Conclusions: The MNA is considerably useful in providing a comprehensive assessment of nutritional status in elderly, community-living Japanese. However, larger-scale epidemiological studies are needed to determine the utility and the appropriate cut-off point of the MNA as a screen for risk of malnutrition.
\end{abstract}

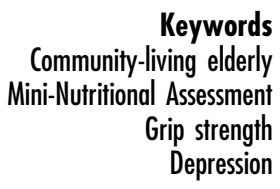

The proportion of elderly people is increasing markedly around the world. In Japan today, $22 \cdot 1 \%$ of the total population is $\geq 65$ years of age, and this statistic is estimated to exceed $33.7 \%$ in 2035 . Moreover, the population of very old people (aged $\geq 75$ years) is expanding to $10 \cdot 2 \%{ }^{(1)}$. This demographic shift will change healthcare problems, and continued good health of the elderly population will remain a major challenge in public health $^{(2)}$. Malnutrition is a common health problem in the elderly, causing a decline in the quality of life. Particularly in community-living elderly, chronic malnutrition is a widespread, but often unrecognized, problem that goes untreated. The prevalence of risk of malnutrition (RM) in the elderly living at home is 24 (SD $0 \cdot 4) \%$ in a previous review $^{(3)}$. Reports suggest that $30 \%$ of Japanese community-living elderly individuals are at RM, and 50\% have multiple health problems requiring medical treatment ${ }^{(4)}$. Increasing health-care expenditures among the elderly is also becoming a serious global socio-economic problem. The assessment of malnutrition risk among the community-living elderly is important for early intervention and prevention, which may contribute to the improvement of the social economy.

Nutritional status in the elderly is influenced by various factors, including physical and psychosocial factors ${ }^{(5,6)}$. Traditional clinically available nutrition-screening tools lack sensitivity and specificity; therefore, to properly evaluate elderly nutritional status, comprehensive assessment tools 
are needed ${ }^{(7)}$. The Mini-Nutritional Assessment (MNA) has been recognized as a comprehensive nutritional assessment tool to screen for at-risk elderly in Europe and the USA. It comprises a matrix of eighteen assessment items in four parts: anthropometric, general, dietary and self-assessment, and has a high predictive value for morbidity and mortality ${ }^{(3)}$. However, the applicability of the MNA has not been proven in Asian populations, whose cultural and dietary habits differ from those of Western populations ${ }^{(8)}$. Recent reports of MNA use in Asian and African populations have yielded conflicting results regarding its utility as a valid indicator of nutritional status ${ }^{(9-12)}$. Discrepant reports in previous studies may be the result of differences in regional culture, physical constitution and economical situation. In addition, the applicability of the MNA for the assessment of community-living elderly individuals has not been investigated sufficiently, particularly in Asian populations. The MNA, which does not require blood tests or assessment by a medical specialist, may be suitable and useful for assessing community-living elderly individuals. Therefore, the applicability of MNA for assessing the Asian community should be investigated.

The aims of the present study were: (i) to examine the utility of MNA in assessing comprehensive nutritional status in community-living elderly Japanese; and (ii) to identify mutable and relative factors with nutritional status as the foundation for preventive intervention in Japan.

\section{Methods}

\section{Subject and settings}

The present cross-sectional study was conducted in August 2007 and August-September 2008 at the Toshima elder-care facility in Tokyo, Japan. We recruited individuals by announcing the study in the Toshima community magazine and on posters displayed in the community's welfare facilities. Inclusion criteria were as follows: (i) elderly individuals (at least 65 years of age) living in the community; (ii) ability to stand unassisted; (iii) ability to communicate with the investigator; and (iv) no presence of a pacemaker or other metal surgical implant. All participants received a document explaining the research and informed consent was given by the 130 enrolled elderly individuals.

An anthropometric survey, biochemical blood examination, assessment of psychosocial and physical functions and questionnaires including basic demographic data (age, sex, marital status, living arrangement, dental status and number of chronic diseases) were used in the present study. All investigations were carried out under the supervision of a physician. Partial data were reported in a pilot study of forty-four individuals, conducted in $2008^{(13)}$. In the present study, excluding the three individuals who had a pacemaker or other metal surgical implant in the body, 130 participants completed an expanded survey and evaluations. The study was approved by the Ethical Committee of Japan Women's University (Tokyo, Japan).

\section{Nutritional status}

Nutritional status was determined by assaying biochemical blood markers and by application of the MNA. All participants were interviewed by educated investigators using the MNA full form, which includes eighteen items (range of scores: 0-30): four anthropometric measurements (BMI, weight loss, mid-arm circumference (AC) and calf circumference (CC)); global evaluation (six questions related to independent living, mobility, medication, presence of psychological stress or acute disease and neuropsychological problems); a dietary questionnaire (six questions related to number of meals, intakes of protein, vegetables, fruit and fluid, and autonomy of feeding); and a subjective assessment (self-perception of health and nutritional status). According to the MNA original manual, the total MNA score is categorized into three groups: well nourished (WN; $>23.5$ points); RM (17.0-23.5 points); and malnourished $(<17 \cdot 0$ points). Participants were categorized into one of two groups: (i) WN ( $>23.5$ points) or (ii) $\mathrm{RM}$ $(\leq 23.5$ points), because the number of malnourished participants $(<17 \cdot 0$ points $)$ was too small $\left(\begin{array}{ll}n & 3\end{array}\right)$ for statistical analyses. All measurements were compared between the WN and RM groups to examine the effectiveness of the $23 \cdot 5$ cut-off value.

\section{Examination of biochemical blood markers}

Fasting blood samples were collected $3 \mathrm{~h}$ after a meal. Determinations of serum total protein (biuret assay), albumin (BCG assay), total cholesterol (cholesterol oxidase assay), HDL cholesterol (direct enzyme assay), TAG (free glycerol enzyme assay), aspartate aminotransferase (enzyme assay), alanine aminotransferase (enzyme assay), $\mathrm{Hb}$ (SLS-Hb assay), cholinesterase activity ( $p$-hydroxybenzoyl choline assay) and C-reactive protein (latex agglutination assay) were measured using an autoanalyser (Hitachi 7600; Hitachi, Tokyo, Japan). Blood samples were collected in EDTA tubes and cell numbers were measured with an autoanalyser (SE-9000; Sysmex, Kobe, Japan). All biochemical markers were measured by contract arrangement with Falco Biosystems Ltd. (Kyoto, Japan).

\section{Antbropometric measurements}

BMI $\left(\mathrm{kg} / \mathrm{m}^{2}\right)$ was measured using an Innerscan BC-610weight scale (Tanita, Tokyo, Japan). AC, CC, triceps skinfold thickness (TSF) and subscapular skinfold thickness (SSF) were measured by an experienced investigator using insertape and an adipometer (Abbott Japan, Tokyo, Japan). Mid-arm muscle area (AMA) was calculated using the formula: $\mathrm{AMA}=[\mathrm{AC}-(\mathrm{TSF} \cdot \pi)]^{2} /(4 \cdot \pi)$. Measured values of $\mathrm{CC}$ and $\mathrm{AC}$ were applied to the MNA assessment. 


\section{Psychosocial functions}

Basic demographic data, including age, gender, marital status, living arrangement, underlying diseases and denture use, were surveyed by a descriptive questionnaire. The Tokyo Metropolitan Institute of Gerontology Index of Competence (TMIG-IC) was used to evaluate higher-level functional capacity, including instrumental activity of daily living based on Lawton's, as described previously ${ }^{(14)}$. It consists of thirteen questions (range of scores: $0 \cdot 0-13 \cdot 0$ ) following three subscales: instrumental self-maintenance (five items: going out using public transportation, shopping, preparing meals, paying bills and depositing at a bank); intellectual activity (four items: writing pensionary documents, reading newspapers, magazines and books and interest in health information); and social role (four items: visiting friend's houses, counselling family and friends, visiting sick friends, talking with young people). For the total and each subscale score, a greater score indicates greater capacity.

Depression was evaluated using a Geriatric Depression Scale-short form (GDS-15), a validated and reliable selfreport scale that detects depressive symptoms in elderly people. The GDS-15 consists of fifteen questions (range of scores: $0 \cdot 0-15 \cdot 0)$ with higher scores indicating more symptoms of depression, with a cut-off score of $6 \cdot 0$ indicating depression ${ }^{(15)}$.

\section{Pbysical function}

Physical function was measured by muscle strength (grip strength) and mobility (usual and maximum speeds of $5 \mathrm{~m}$ walks). Grip strength was assessed using a digital squeeze dynamometer (T.K.K. 5401; Takei Scientific Instruments, Niigata, Japan), with mean measurements derived from duplicate tests on both the left and right hands. In the $5 \mathrm{~m}$ walking speed tests, both usual and maximum voluntary speeds were measured twice; faster times were applied to the data analysis. All participants underwent blood pressure monitoring before assessment of physical function. Participants with systolic blood pressure $>180 \mathrm{mmHg}$ or diastolic blood pressure $>110 \mathrm{mmHg}$ were not tested for grip strength.

\section{Statistical analysis}

All statistical calculations were carried out with the Statistical Package for the Social Sciences statistical software package version 16.0 (SPSS Inc., Tokyo, Japan). Mean and SD values were used as descriptive statistics; the Kolmogorov-Smirnov test for normality was applied. The correlation between the MNA score and variable measurements (basic characteristics, anthropometric measurements, biochemical blood markers and psychosocial functions) was analysed by Spearman's rank correlation test. The partial correlation analysis (with two control variables: sex and age) was used to assess the relationship between physical function and MNA score, because physical function decreases with age and is greater in men ${ }^{(16,17)}$. The Mann-Whitney $U$ test and the $\chi^{2}$ test were used to compare the two MNA score groups (WN $v$. RM). Stepwise linear multiple regression analysis was carried out, which included basic demographic factors, physical functions and psychosocial functions to explain independent factors of the MNA. Although the study population included a majority of women, the analytical results by division of sex were similar in both sexes for all parameters. Therefore, the results of the present study are not divided by sex. A value of $P<0.05$ was considered significant.

\section{Results}

\section{Subject demographics}

The 130 elderly participants included twenty-six men and 104 women; details are presented in Table 1 . The mean age was $76 \cdot 6$ (SD $6 \cdot 3$ ) years. Seventy-nine participants $(60 \cdot 6 \%)$ aged $\geq 75$ years were described as very old and others as young old. Thirty-six participants $(27 \cdot 7 \%)$ were living alone, fifty-eight $(44.6 \%)$ were living with a spouse and 101 were using dentures $(77 \cdot 7 \%)$. A total of 109 participants $(83 \cdot 8 \%)$ suffered from one or more chronic diseases.

\section{Mini-Nutritional Assessment score and correlation with nutritional, physical and psychological functions}

The mean MNA score was $25 \cdot 8$ (SD 3.3). According to the MNA score, twenty-seven (20.8\%) participants were assessed to be at RM, including three with an MNA score $<17 \cdot 5$. A total of 103 participants $(79 \cdot 2 \%)$ were categorized as WN. The mean age and number of very old participants were higher in the RM group (85.2\%) compared with the WN group $(54.4 \%)$. Participants having three or more chronic diseases were significantly over-represented in the RM group (51.9\%) compared with the WN group (22.3\%). There were no differences between the two groups in living arrangement, marital status and dental status.

Conventional evaluation of nutritional status with biochemical blood markers (red blood cells, Hb, total protein, albumin, cholinesterase and TLC) and anthropometric measurements (TSF, SSF and AMA) was significantly correlated with MNA score (Table 2). The mean values of these biochemical blood markers and anthropometric measurements (TSF, SSF and AMA) were significantly lower in the RM group compared with the WN group. BMI, AC and CC were excluded from the analysis because these metrics were contained within the MNA evaluation.

With regard to physical functions, only grip strength showed a significant correlation with MNA score $(r=0 \cdot 467$, $P<0 \cdot 001)$, adjusted by sex and age. In comparison with the WN group, grip strength among participants at RM was significantly lower $(P=0 \cdot 009)$, and walking speeds, both usual and maximum, were also slower (usual: $P=0 \cdot 002$; 
Table 1 Basic characteristics and MNA score of the community-dwelling elderly Japanese

\begin{tabular}{|c|c|c|c|c|c|c|c|c|c|c|}
\hline \multirow[b]{2}{*}{ Variable } & \multicolumn{3}{|c|}{ All participants ( $n$ 130) } & \multicolumn{3}{|c|}{ Risk of malnutrition (MNA $\leq 23 \cdot 5 ; n 27$ ) } & \multicolumn{3}{|c|}{ Well nourished (MNA > 23.5; $n$ 103) } & \multirow[b]{2}{*}{$P$ value } \\
\hline & Mean or $n$ & $\%$ or SD & Range & Mean or $n$ & $\%$ or SD & Range & Mean or $n$ & $\%$ or SD & Range & \\
\hline Age $\left(\right.$ years) ${ }^{*}$ & $76 \cdot 6$ & $6 \cdot 3$ & $65-94$ & $78 \cdot 6$ & $5 \cdot 8$ & $65-91$ & $76 \cdot 1$ & $6 \cdot 4$ & $65-94$ & $0.043+$ \\
\hline 65-74 (young old) & 51 & $39 \cdot 4$ & - & 4 & $14 \cdot 8$ & - & 47 & $45 \cdot 6$ & - & $0 \cdot 004 \ddagger$ \\
\hline$\geq 75$ (very old) & 79 & $60 \cdot 6$ & - & 23 & $85 \cdot 2$ & - & 66 & $54 \cdot 4$ & - & \\
\hline Sex & & & & & & & & & & $0 \cdot 116 \ddagger$ \\
\hline Male & 26 & $20 \cdot 0$ & - & 7 & $25 \cdot 9$ & - & 19 & $18 \cdot 4$ & - & \\
\hline Female & 104 & $80 \cdot 0$ & - & 20 & $74 \cdot 1$ & - & 84 & $81 \cdot 6$ & - & \\
\hline BMI $\left(\mathrm{kg} / \mathrm{m}^{2}\right)^{*}$ & $22 \cdot 8$ & $3 \cdot 6$ & $16 \cdot 5-35 \cdot 7$ & $19 \cdot 4$ & $2 \cdot 3$ & $16 \cdot 5-25 \cdot 3$ & $23 \cdot 8$ & $3 \cdot 3$ & $17 \cdot 5-35 \cdot 7$ & $<0.001 t$ \\
\hline Living arrangement & & & & & & & & & & $0 \cdot 074 \ddagger$ \\
\hline Living alone & 36 & $27 \cdot 7$ & - & 7 & $25 \cdot 9$ & - & 29 & $28 \cdot 2$ & - & \\
\hline Living with a spouse & 58 & $44 \cdot 6$ & - & 8 & $29 \cdot 6$ & - & 50 & $48 \cdot 5$ & - & \\
\hline Living together, except with a spouse & 36 & $27 \cdot 7$ & - & 12 & $44 \cdot 4$ & - & 24 & $23 \cdot 3$ & - & \\
\hline Marital status & & & & & & & & & & $0 \cdot 087 \ddagger$ \\
\hline Married & 58 & $44 \cdot 6$ & - & 8 & $29 \cdot 6$ & - & 50 & $48 \cdot 5$ & - & \\
\hline Not married & 72 & $55 \cdot 4$ & - & 19 & $70 \cdot 4$ & - & 53 & $55 \cdot 4$ & - & \\
\hline Dental status & & & & & & & & & & $0.437 \ddagger$ \\
\hline Natural dentition & 29 & $22 \cdot 3$ & - & 4 & $14 \cdot 8$ & - & 25 & $24 \cdot 3$ & - & \\
\hline Partial or complete dentures & 101 & $77 \cdot 7$ & - & 23 & $85 \cdot 2$ & - & 78 & $75 \cdot 7$ & - & \\
\hline Number of chronic diseases & & & & & & & & & & $0 \cdot 017 \ddagger$ \\
\hline 1 or 2 & 72 & $55 \cdot 4$ & - & 13 & $48 \cdot 1$ & - & 80 & $77 \cdot 7$ & - & \\
\hline$\geq 3$ & 37 & $28 \cdot 5$ & - & 14 & $51 \cdot 9$ & - & 23 & $22 \cdot 3$ & - & \\
\hline MNA score* & $25 \cdot 8$ & $3 \cdot 3$ & $13 \cdot 0-30 \cdot 0$ & $20 \cdot 5$ & $2 \cdot 9$ & $13 \cdot 0-23 \cdot 5$ & $27 \cdot 1$ & $1 \cdot 5$ & $24 \cdot 0-30 \cdot 0$ & $<0.001 \dagger$ \\
\hline
\end{tabular}

MNA, Mini-Nutritional Assessment.

*Data are presented as mean, SD and range.

$+P$ value obtained using the Mann-Whitney $U$ test.

$\ddagger P$ value obtained using the $\chi^{2}$ test. 
Table 2 Correlation of variable factors with MNA score and comparison between MNA groups of the community-dwelling elderly Japanese

\begin{tabular}{|c|c|c|c|c|c|c|c|c|c|}
\hline & \multicolumn{2}{|c|}{ All participants } & \multirow[b]{2}{*}{$r$} & \multirow[b]{2}{*}{$P$ value ${ }^{*}$} & \multicolumn{2}{|c|}{$\begin{array}{l}\text { Risk of malnutrition } \\
(\mathrm{MNA} \leq 23 \cdot 5 ; n 27)\end{array}$} & \multicolumn{2}{|c|}{$\begin{array}{c}\text { Well nourished } \\
(\mathrm{MNA}>23.5 ; n \text { 103) }\end{array}$} & \multirow[b]{2}{*}{$P$ valuet } \\
\hline & Mean & SD & & & Mean & SD & Mean & SD & \\
\hline \multicolumn{10}{|l|}{ Biochemical markers } \\
\hline WBC $\left(10^{2} / \mathrm{ml}\right)$ & $59 \cdot 0$ & $15 \cdot 0$ & 0.022 & $0 \cdot 804$ & $57 \cdot 0$ & $15 \cdot 0$ & $59 \cdot 0$ & $15 \cdot 0$ & $0 \cdot 516$ \\
\hline $\mathrm{RBC}\left(10^{4} / \mathrm{ml}\right)$ & $420 \cdot 0$ & $40 \cdot 0$ & $0 \cdot 187$ & 0.034 & $407 \cdot 0$ & $48 \cdot 0$ & $423 \cdot 0$ & $37 \cdot 0$ & 0.043 \\
\hline $\mathrm{Hb}(\mathrm{g} / \mathrm{dl})$ & $12 \cdot 9$ & $1 \cdot 3$ & 0.232 & 0.008 & $12 \cdot 4$ & $1 \cdot 3$ & $13 \cdot 0$ & $1 \cdot 2$ & $0 \cdot 011$ \\
\hline Total protein (g/dl) & $7 \cdot 4$ & 0.4 & $0 \cdot 107$ & 0.233 & $7 \cdot 3$ & 0.5 & $7 \cdot 4$ & $0 \cdot 4$ & 0.026 \\
\hline Albumin $(\mathrm{g} / \mathrm{dl})$ & $4 \cdot 4$ & $0 \cdot 3$ & 0.256 & 0.004 & $4 \cdot 2$ & $0 \cdot 3$ & $4 \cdot 5$ & $0 \cdot 2$ & $<0.001$ \\
\hline AST (IU/I) & $24 \cdot 0$ & $8 \cdot 0$ & 0.072 & $0 \cdot 417$ & $24 \cdot 0$ & $6 \cdot 0$ & $24 \cdot 0$ & $9 \cdot 0$ & 0.333 \\
\hline ALT (IU/I) & $19 \cdot 0$ & $10 \cdot 0$ & $0 \cdot 181$ & 0.041 & $17 \cdot 0$ & $8 \cdot 0$ & $19 \cdot 0$ & $10 \cdot 0$ & $0 \cdot 238$ \\
\hline Cholinesterase (IU/I) & $305 \cdot 0$ & $65 \cdot 0$ & 0.380 & $<0.001$ & $267 \cdot 0$ & $58 \cdot 0$ & $315 \cdot 0$ & $63 \cdot 0$ & $<0.001$ \\
\hline Total cholesterol (mg/dl) & $216 \cdot 0$ & $34 \cdot 0$ & -0.003 & 0.972 & $213 \cdot 0$ & $37 \cdot 0$ & $217 \cdot 0$ & $34 \cdot 0$ & 0.569 \\
\hline HDL cholesterol (mg/dl) & $70 \cdot 0$ & $20 \cdot 0$ & 0.007 & 0.935 & $71 \cdot 0$ & $24 \cdot 0$ & $69 \cdot 0$ & $19 \cdot 0$ & 0.935 \\
\hline LDL cholesterol (mg/dl) & $129 \cdot 0$ & $30 \cdot 0$ & -0.008 & 0.929 & $126 \cdot 0$ & $32 \cdot 0$ & $130 \cdot 0$ & $29 \cdot 0$ & $0 \cdot 438$ \\
\hline $\operatorname{TLC}(/ \mu \mathrm{l})$ & $1937 \cdot 0$ & $583 \cdot 0$ & 0.247 & 0.005 & $1687 \cdot 0$ & $585 \cdot 0$ & $2003 \cdot 0$ & $568 \cdot 0$ & 0.010 \\
\hline \multicolumn{10}{|l|}{ Anthropometric measurements } \\
\hline $\operatorname{TSF}(\mathrm{mm})$ & $15 \cdot 0$ & $5 \cdot 7$ & 0.369 & $<0.001$ & $10 \cdot 8$ & $5 \cdot 3$ & $15 \cdot 9$ & $5 \cdot 3$ & $<0.001$ \\
\hline $\mathrm{SSF}(\mathrm{mm})$ & $15 \cdot 5$ & $6 \cdot 2$ & 0.371 & $<0.001$ & $11 \cdot 1$ & $5 \cdot 1$ & $16 \cdot 4$ & $6 \cdot 0$ & $<0.001$ \\
\hline AMA $\left(\mathrm{cm}^{2}\right)$ & $39 \cdot 9$ & $7 \cdot 9$ & 0.527 & $<0.001$ & $32 \cdot 8$ & $6 \cdot 5$ & $41 \cdot 7$ & $7 \cdot 2$ & $<0.001$ \\
\hline \multicolumn{10}{|l|}{ Physical function } \\
\hline Grip strength (kg) & $22 \cdot 1$ & $7 \cdot 1$ & 0.467 & $<0.001$ & $18 \cdot 9$ & $7 \cdot 4$ & $22 \cdot 9$ & $6 \cdot 8$ & 0.009 \\
\hline Usual walking speed (s) & $4 \cdot 4$ & $1 \cdot 4$ & -0.156 & 0.084 & $5 \cdot 1$ & $1 \cdot 8$ & $4 \cdot 3$ & $1 \cdot 2$ & 0.002 \\
\hline Maximum walking speed (s) & $3 \cdot 3$ & 0.8 & -0.092 & 0.309 & $3 \cdot 6$ & $1 \cdot 1$ & $3 \cdot 2$ & $0 \cdot 7$ & 0.033 \\
\hline TMIG-IC & $11 \cdot 8$ & $1 \cdot 8$ & 0.530 & $<0.001$ & $10 \cdot 9$ & $2 \cdot 0$ & $12 \cdot 0$ & $1 \cdot 6$ & 0.001 \\
\hline GDS score & $3 \cdot 4$ & $3 \cdot 3$ & -0.297 & 0.001 & $6 \cdot 2$ & $4 \cdot 1$ & $2 \cdot 7$ & $2 \cdot 7$ & $<0.001$ \\
\hline
\end{tabular}

MNA, Mini-Nutritional Assessment; WBC, white blood cell; RBC, red blood cell; AST, aspartate aminotransferase; ALT, alanine aminotransferase; TLC, total lymphocyte count; TSF, triceps skinfolds; SSF, subscapular skinfold; AMA, mid-arm muscle area; TMIG-IC, Tokyo Metropolitan Institute of Gerontology Index of Competence; GDS, Geriatric Depression Scale.

${ }^{\star} P$ value obtained using Peason's correlation coefficient, but physical functions were analysed using partial correlation (control variables: sex and age).

$+P$ value obtained using the Mann-Whitney $U$ test.

Table 3 Stepwise multiple regression model of variables associated with MNA score of the community-dwelling elderly Japanese

\begin{tabular}{|c|c|c|c|}
\hline Variable* $^{*}$ & $\beta$ & $t$ & $P$ \\
\hline \multicolumn{4}{|c|}{ All participants ( $n 130$, adjusted $R^{2}=0.444, P<0.001$ ) } \\
\hline Grip strength & $0 \cdot 627$ & $5 \cdot 284$ & $<0.001$ \\
\hline GDS score & -0.346 & $-4 \cdot 664$ & $<0.001$ \\
\hline Marital statust & -0.211 & $-2 \cdot 753$ & 0.008 \\
\hline Maximum walking speed (s) & $0 \cdot 327$ & $2 \cdot 463$ & 0.015 \\
\hline \multicolumn{4}{|c|}{ Young old ( $n 51$, adjusted $\left.R^{2}=0.463, P<0.001\right)$} \\
\hline Number of chronic diseases & -0.355 & $-2 \cdot 823$ & 0.007 \\
\hline Marital statust & -0.305 & $-2 \cdot 666$ & 0.011 \\
\hline Grip strength & 0.278 & $2 \cdot 005$ & 0.051 \\
\hline Denture status $\ddagger$ & $0 \cdot 192$ & $1 \cdot 623$ & $0 \cdot 112$ \\
\hline \multicolumn{4}{|c|}{ Very old $\left(n 79\right.$, adjusted $\left.R^{2}=0.390, P<0.001\right)$} \\
\hline Grip strength & 0.627 & $4 \cdot 478$ & $<0.001$ \\
\hline GDS score & -0.326 & $-3 \cdot 372$ & 0.001 \\
\hline
\end{tabular}

MNA, Mini-Nutritional Assessment; GDS, Geriatric Depression Scale; TMIG-IC, Tokyo Metropolitan Institute of Gerontology Index of Competence.

*Independent variables used basic demographic factors (age, sex, living arrangement, marital status, dental status and number of chronic diseases), physical functions (grip strength, usual and maximum walking speeds) and psychosocial functions (TMIG-IC and GDS) for stepwise multiple regression analysis.

tHaving a marital partner is correlated with higher MNA scores.

‡Denture use is correlated with lower MNA scores. 
(65-74 years) and the very old ( $\geq 75$ years). Among the young old, number of chronic diseases, having a marital partner, grip strength and denture use were considered as explanatory factors (adjusted $R^{2}=0.463, \quad P<0.001$ ). Among the very old, only two factors, grip strength and GDS score, were considered as MNA score explanatory factors (adjusted $R^{2}=0 \cdot 390, P<0 \cdot 001$ ).

\section{Discussions}

Malnutrition, a common health problem in the elderly, leads to poor outcomes such as functional decline, frailty, declining quality of life and higher mortality. The assessment of RM among the community-living elderly is important for early intervention and prevention to improve the quality of life. MNA is recognized as a wellvalidated nutritional assessment tool for the elderly in Europe and the USA and is used to screen for early $\mathrm{RM}^{(3)}$. Despite the high prevalence of malnutrition in the elderly, there is no standardized method for comprehensive nutritional assessment of the elderly in Japan and in other Asian countries. Serum albumin and BMI have been used frequently as markers of malnutrition in Japan ${ }^{(4,11)}$. However, serum albumin levels are responsive to many other factors, such as inflammatory disease and drug or hormone therapy ${ }^{(18)}$, and is therefore inappropriate for diagnosis of malnutrition in the elderly. Elderly people with normal BMI can be malnourished, because nutritional status in the elderly is influenced by various factors including physical and psychosocial factors that influence the quality of life ${ }^{(5,6)}$. Therefore, elderly individuals must be screened early for RM by assessing comprehensive factors.

In the present study, MNA score correlated with existing nutritional markers, including biomarkers and anthropometric measurements. MNA score also correlated with physical and psychosocial functions, suggesting that the MNA provides a comprehensive assessment of nutritional status in community-living elderly in Japan, as well as in Europe and the USA. Traditional nutritional markers, such as serum albumin, indicated low $r$ values when correlating with MNA score, although it is possible that our participants were relatively healthy. The purpose of the study was to evaluate the utility of the MNA as a comprehensive nutritional evaluation tool; we thought, therefore, that the low $r$ values were an acceptable result.

The MNA as well as drawing of blood can be performed without a medical specialist; it is easily applicatory in the case of carrying out nutritional assessment of community-living elderly. In Japanese clinical practice, subjective global assessment (SGA) is used to evaluate comprehensive nutritional status in patients, including the elderly $^{(19)}$. The geriatric nutritional risk index (GNRI) is a nutritional screening tool for elderly Europeans that is based on albumin and BMI values ${ }^{(20)}$. The SGA and GNRI require a medical specialist or blood sampling, making these assessments unsuitable for community-living individuals; therefore, it is important that these assessments correlate well with the MNA (SGA: $r=0 \cdot 590, P<0 \cdot 001$, GNRI: $r=0 \cdot 373, P<0 \cdot 001$, data not shown). Anthropometric measurements are commonly used to determine a person's percentage of body fat or body composition as a reflection of nutritional function ${ }^{(21)}$. TSF, SSF and AMA also correlated with MNA score. These correlation data supported the utility of the MNA as a comprehensive nutritional status assessment tool.

However, Asians have different regional dietary habits and physical constitutions; some reports have suggested that the MNA might not be applicable to Asians, and that the cut-off point of the assessment may need to be changed $^{(12)}$. The present study could not prove the screening fidelity of the MNA for RM in the elderly Japanese, because we did not examine the cut-off point. Further testing is required to properly define the utility of the MNA and the appropriate cut-off point in Japanese elderly.

Multiple regression analyses revealed a number of factors that independently affect the MNA score, including (in descending order of importance) grip strength, GDS depression status, marital status and maximum walking speeds. Our findings were similar to those reported previously ${ }^{(5,6,16)}$, but more strongly indicated that grip strength is most strongly correlated with RM in the community-living elderly. Although the question for neuropsychological status was included in the MNA, no participant was judged to have neuropsychological abnormalities, which indicates that the relationship between GDS and MNA was strong.

We noted that correlated factors differed between young old and very old participants. In the very old, only two factors, grip strength and depressive status, were identified as having a strong correlation with MNA score. In the young old, chronic diseases and marital status were more influential than grip strength, and depression status was not a significant factor. Weakness of grip strength and depression are known to occur easily with age ${ }^{(22)}$, and may explain the different results between age groups. Our results indicated that grip strength and depression became more critical factors in nutritional status with age; thus, more flexible and comprehensive nutritional intervention is needed for individuals of advanced age. Further longitudinal studies are needed to determine causality in the relationship between nutritional status, grip strength and depression.

In the present cross-sectional study of communityliving Japanese elderly, grip strength and depressive function were associated with nutritional status as assessed by the MNA. With over $20 \%$ of elderly at RM, it is important to increase public awareness of nutritional assessments that account for mental and physical functions in the community-living elderly. In addition, although we confirmed that the analysis of the 127 subjects was unchanged by our exemption of the three subjects judged 'malnourished (MNA < 17)', our results emphasize the need to undertake 
large-scale epidemiological studies to properly examine the screening capabilities and appropriate cut-off points for the three MNA classifications.

\section{Acknowledgements}

The present study was supported by the Ministry of Education, Culture, Sports, Science and Technology, outlays for promoting science and technology, the Multi Career Path Support Model for Female Researchers of Japan Women's University and by Grant-in-Aid for Scientific Research (C) 20300247, Ministry of Education, Science, and Culture, Japan (to K.S.). The authors have no conflict of interest to declare. The authors contributed as follows. Conception and design of study: T.K., R.H. and K.S. Acquisition of data: T.K., R.H., H.Y., Y.O., M.S., M.N., E.F. and K.S. Analysis of data: T.K. and R.H. Statistical expertise: K.K. Drafting of manuscript: T.K. and K.S. We thank all participants and officers in the elder-care facility of Toshima for their cooperation in the study, and technical assistance provided by Mrs Y. Inoue, Mrs M. Tashiro and members of the Clinical Nutrition Laboratory, Japan Women's University.

\section{References}

1. Japanese Cabinet Office (2009) Aging Society White Paper. Tokyo: Cabinet Office.

2. Inouye SK, Studenski S, Tinetti ME et al. (2007) Geriatric syndromes: clinical, research, and policy implications of a core geriatric concept. J Am Geriatr Soc 55, 780-791.

3. Guigoz Y, Vellas B \& Garry PJ (1996) Assessing the nutritional status of the elderly: the Mini Nutritional Assessment as part of the geriatric evaluation. Nutr Rev 54, S59-S65.

4. Ministry of Health, Labour and Welfare (2006) National Livelihood Survey. Tokyo: MHLW.

5. Goodwin JS (1989) Social, psychological and physical factors affecting the nutritional status of elderly subjects: separating cause and effect. Am J Clin Nutr 50, 1201-1209.

6. Cabrera MA, Mesas AE, Garcia AR et al. (2007) Malnutrition and depression among community-dwelling elderly people. Am Med Dir Assoc 8, 582-584.
7. Gariballa SE \& Sinclair AJ (1998) Nutrition, ageing and ill health. BrJ Nutr 80, 7-23.

8. Chumlea WC (2006) Is the MNA valid in different populations and across practice settings? J Nutr Health Aging 10, 524-527.

9. Charlton KE, Kolbe-Alexander TL \& Nel JH (2005) Development of a novel nutrition screening tool for use in elderly South Africans. Public Health Nutr 8, 468-479.

10. Lei Z, Qingyi D, Feng G et al. (2009) Clinical study of mininutritional assessment for older Chinese inpatients. J Nutr Health Aging 13, 871-875.

11. Izawa S, Kuzuya M, Okada K et al. (2006) The nutritional status of frail elderly with care needs according to the mini-nutritional assessment. Clin Nutr 25, 962-967.

12. Kuzuya M, Kanda S, Koike T et al. (2005) Evaluation of Mini-Nutritional Assessment for Japanese frail elderly. Nutrition 21, 498-503.

13. Hirasawa R, Kaburagi T, Yoshino H et al. (2009) Analysis of nutritional status and relative factors of malnutrition in community-dwelling Japanese elderly. J Metab Clin Nutr 12, 137-147.

14. Koyano W, Shibata H, Nakazato K et al. (1991) Measurement of competence: reliability and validity of the TMIG Index of Competence. Arch Gerontol Geriatr 13, 103-116.

15. Yesavage JA (1988) Geriatric depression scale. Psychopharmacol Bull 24, 709-711.

16. Shinkai S, Watanabe S, Kumagai S et al. (2000) Walking speed as a good predictor for the onset of functional dependence in a Japanese rural community population. Age Ageing 29, 441-446.

17. Shechtman O, Mann WC, Justiss MD et al. (2004) Grip strength in the frail elderly. Am J Phys Med Rehabil 83, 819-826.

18. Kuzuya M, Izawa S, Enoki H et al. (2007) Is serum albumin a good marker for malnutrition in the physically impaired elderly? Clin Nutr 26, 84-90.

19. Wakahara T, Shiraki M, Murase K et al. (2007) Nutritional screening with Subjective Global Assessment predicts hospital stay in patients with digestive diseases. Nutrition 23, 634-639.

20. Cereda E \& Pedrolli C (2009) The geriatric nutritional risk index. Curr Opin Clin Nutr Metab Care 12, 1-7.

21. Omran ML \& Morley JE (2000) Assessment of protein energy malnutrition in older persons, part I: History, examination, body composition, and screening tools. Nutrition 16, 50-63.

22. Chilima DM \& Ismail SJ (2001) Nutrition and handgrip strength of older adults in rural Malawi. Public Health Nutr 4, 11-17. 